\title{
Erratum: Constraining parton distribution functions from neutral current Drell-Yan measurements [Phys. Rev. D 98, 013003 (2018)]
}

\author{
E. Accomando, J. Fiaschi, F. Hautmann, and S. Moretti
}

(Received 19 March 2019; published 10 April 2019)

DOI: 10.1103/PhysRevD.99.079902

The following changes should be noted:

(1) Page 4, end of Sec. II: " $M_{l l}<200 \mathrm{GeV}$ " should be " $M_{l l}<170 \mathrm{GeV".}$

(2) Page 6: "for $M_{l l}<1200 \mathrm{GeV}$ in the CT14NNLO case and for $M_{l l}<1150 \mathrm{GeV}$ in the NNPDF3.1 case." should be "for $M_{l l}<1150 \mathrm{GeV}$ in both the CT14NNLO and NNPDF3.1 cases."

(3) Page 6: "this is true for roughly $M_{l l}<400 \mathrm{GeV}$ " should be "this is true for roughly $M_{l l}<370 \mathrm{GeV}$ ". Furthermore, Figs. 1-5 are updated as follows:
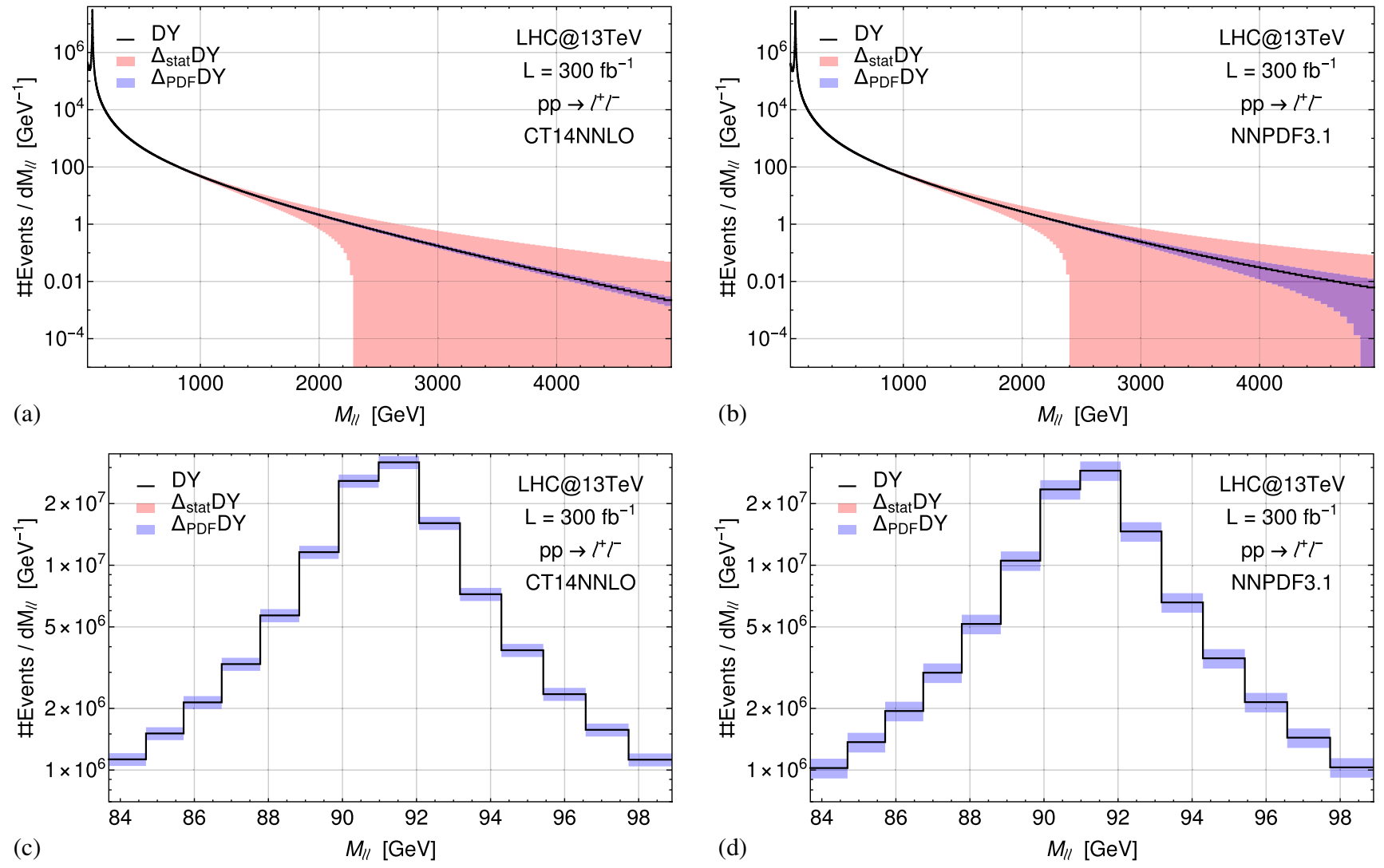

FIG. 1. (a) Expected number of events in the dilepton invariant mass region and their statistical and PDF error obtained with CT14NNLO. (b) Same as (a) obtained with NNPDF3.1. (c) Zoom of (a) around the Z-boson peak. (d) Zoom of (b) around the Zboson peak.

Published by the American Physical Society under the terms of the Creative Commons Attribution 4.0 International license. Further distribution of this work must maintain attribution to the author(s) and the published articles title, journal citation, and DOI. 

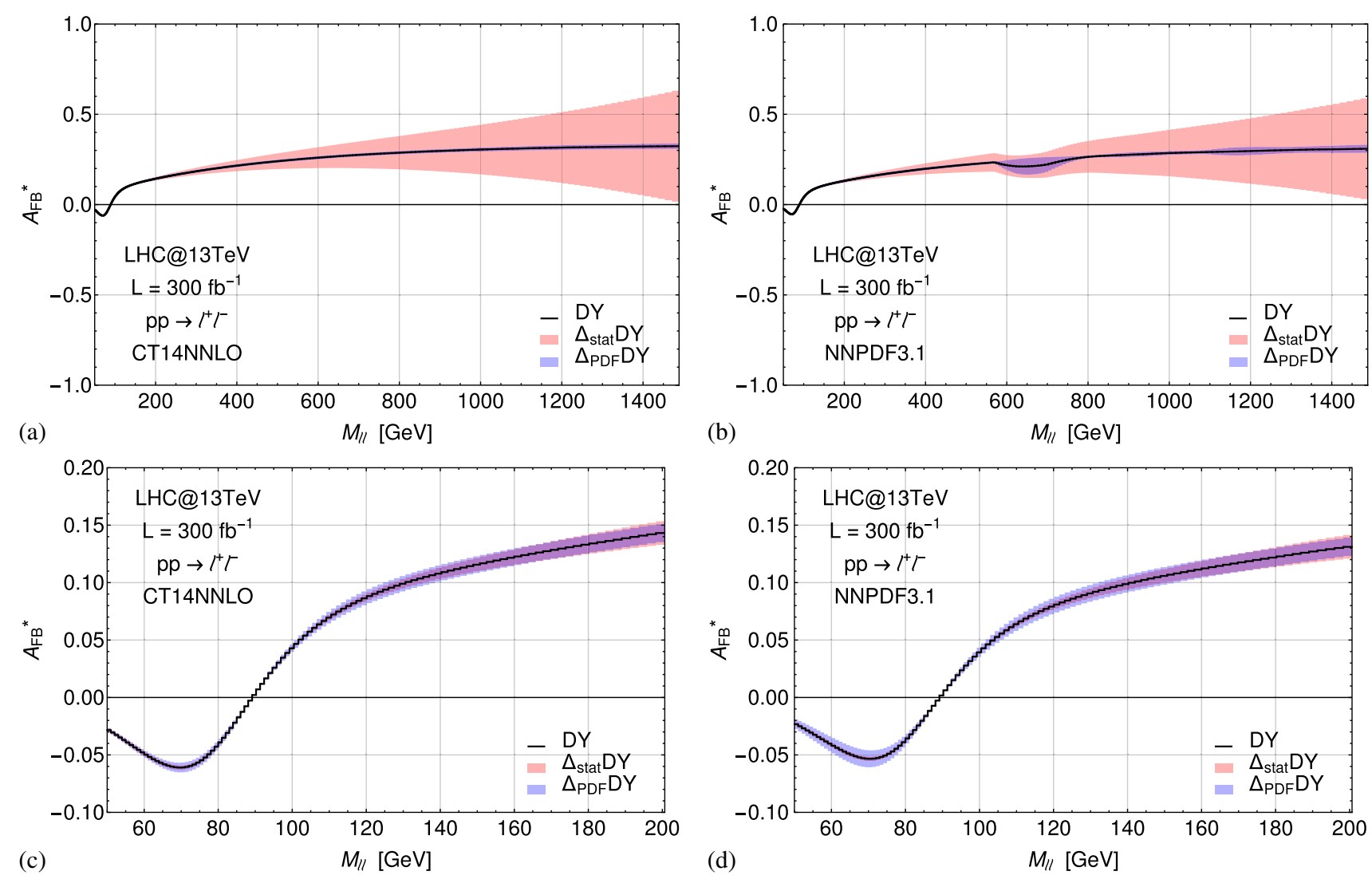

FIG. 2. (a) $A_{\mathrm{FB}}^{*}$ distribution in the dilepton invariant mass including the statistical and PDF errors obtained with CT14NNLO. (b) Same as (a) for the NNPDF3.1 PDF set. (c) Zoom of (a) around the Z-boson peak. (d) Zoom of (b) around the Z-boson peak. 

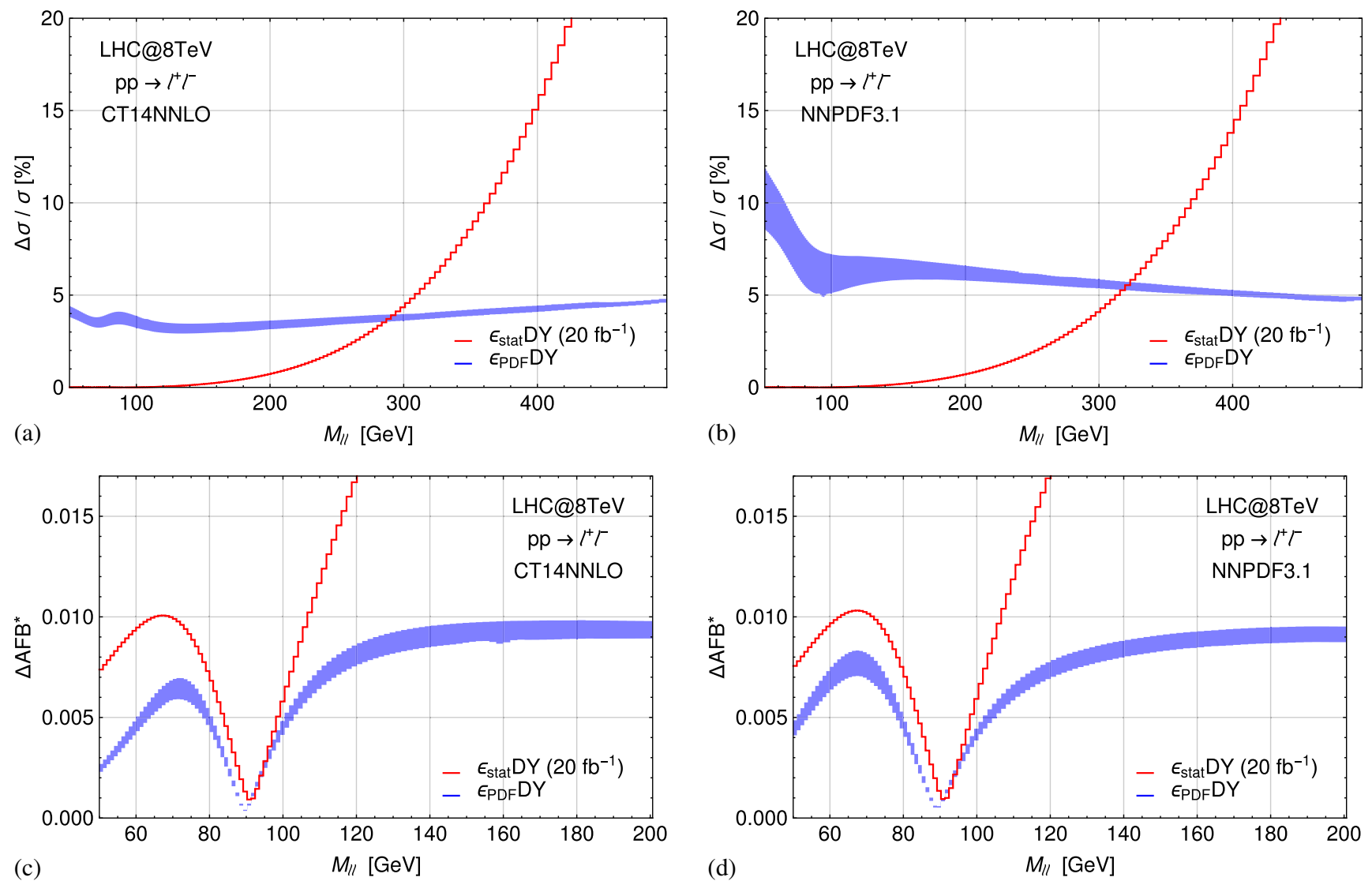

FIG. 3. (a) Relative errors on the differential cross section at the LHC Run-I with $\sqrt{s}=8 \mathrm{TeV}$ and $L=20 \mathrm{fb}^{-1}$, computed with the CT14NNLO PDF set. The red curve represents the statistical error. The blue band has been obtained evaluating the PDF error fixing the factorization/renormalization scale in the interval: $0.5 M_{\ell \ell}<Q<2 M_{\ell \ell}$. (b) Same as (a) for the NNPDF3.1 PDF set. (c) Absolute errors on the reconstructed FB asymmetry at the LHC Run-I, computed with the CT14NNLO PDF set. The red curve represents the statistical error. The blue band refers to the PDF uncertainty. (d) Same as (c) for the NNPDF3.1 PDF set. 

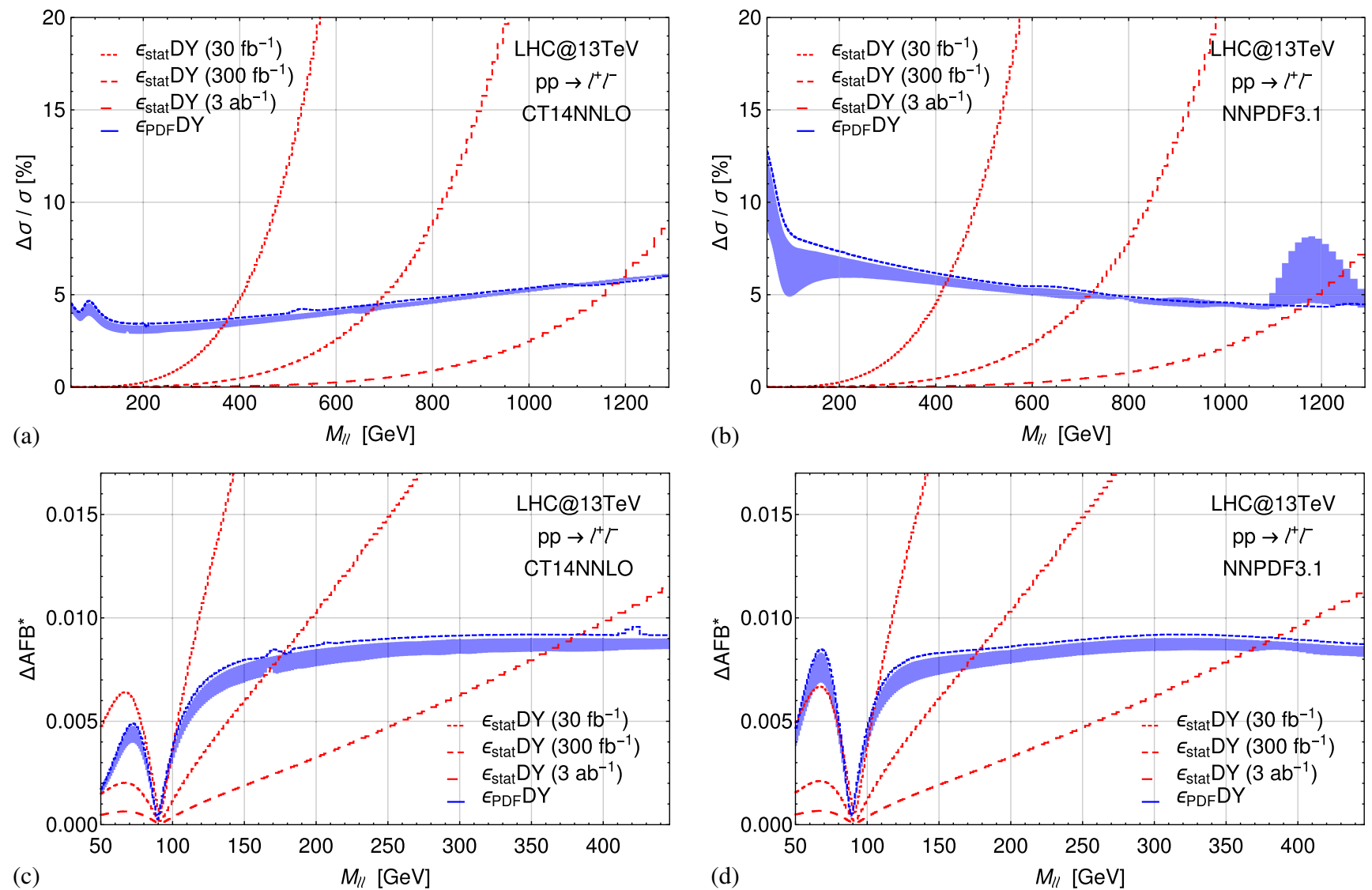

FIG. 4. (a) Relative size of statistical error and PDF uncertainty on the differential cross section obtained with the CT14NNLO set at the $13 \mathrm{TeV}$ LHC. The statistical error is displayed for three different values of the luminosity (see legend). The PDF error band has been obtained evaluating the PDF error fixing the factorization/renormalization scale in the interval: $0.5 M_{\ell \ell}<Q<2 M_{\ell \ell}$, while the dashed blue lines represent the PDF uncertainty obtained fixing $\mu_{F}=\mu_{R}=p_{T}$, with $p_{T}$ the transverse momentum of either lepton in the final state. (b) Same as plot (a) for the NNPDF3.1 PDF set. (c) Absolute value of statistical and PDF uncertainties on the reconstructed $A_{\mathrm{FB}}^{*}$

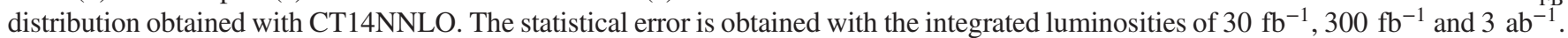
The PDF error bands and lines follow the conventions of plots (a) and (b). (d) Same as (c) for the NNPDF3.1 PDF set. 

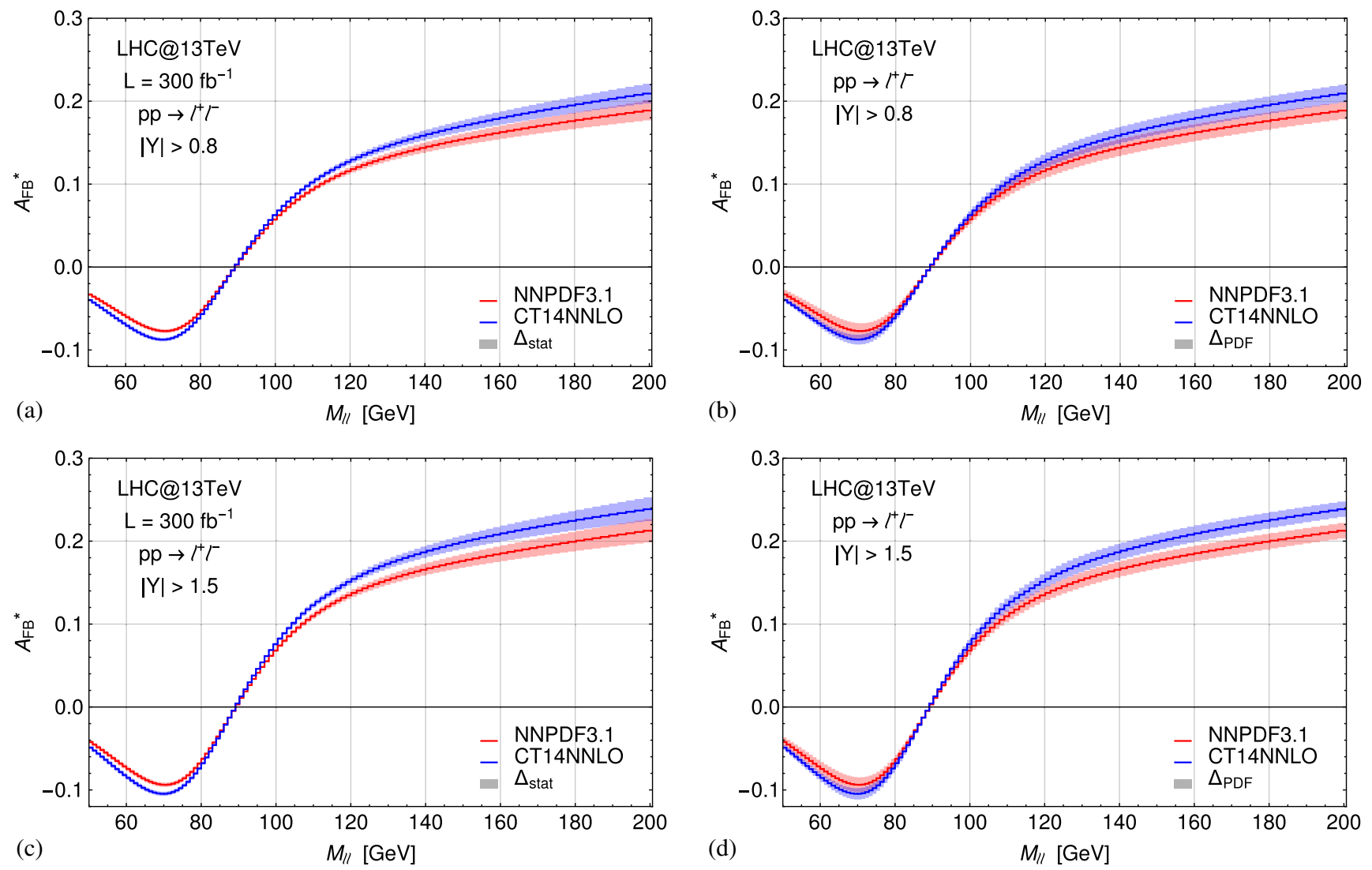

FIG. 5. (a) $A_{\mathrm{FB}}^{*}$ distribution in the invariant mass region around the $Z$ peak at the LHC Run-II with $L=300 \mathrm{fb}^{-1}$, computed with the CT14NNLO and the NNPDF3.1 PDF sets. The dilepton rapidity cut $\left|Y_{\ell \ell}\right|>0.8$ is imposed. The uncertainty band represents the statistical error. (b) Same as (a) with the PDF error band. (c) Same as (a) for the dilepton rapidity cut $\left|Y_{\ell \ell}\right|>1.5$. (d) Same as (b) for the dilepton rapidity cut $\left|Y_{\ell \ell}\right|>1.5$. 